\title{
Contribution of visual and proprioceptive information to the precision of reaching movements
}

\author{
Simona Monaco · Gregory Króliczak • Derek J. Quinlan • \\ Patrizia Fattori · Claudio Galletti · Melvyn A. Goodale • \\ Jody C. Culham
}

Received: 19 August 2009/Accepted: 14 November 2009/Published online: 6 December 2009

(C) Springer-Verlag 2009

\begin{abstract}
Ren et al. (J Neurophysiol 96:1464-1477, 2006) found that saccades to visual targets became less accurate when somatosensory information about hand location was added, suggesting that saccades rely mainly on vision. We conducted two kinematic experiments to examine whether or not reaching movements would also show such strong reliance on vision. In Experiment 1, subjects used their dominant right hand to perform reaches, with or without a delay, to an external visual target or to their own left fingertip positioned either by the experimenter or by the participant. Unlike saccades, reaches became more accurate and precise when proprioceptive information was available. In Experiment 2, subjects reached toward external or bodily targets with differing amounts of visual information. Proprioception improved performance only when vision was limited. These results indicate that the reaching system has a better internal model for limb positions than does the saccade system.
\end{abstract}

Keywords Mutlisensory integration - Vision .

Proprioception $\cdot$ Reaching $\cdot$ Action

S. Monaco $(\bowtie)$ · D. J. Quinlan · M. A. Goodale · J. C. Culham Department of Psychology, The University of Western Ontario, London, ON N6A 5C2, Canada

e-mail: simona.monaco@gmail.com

S. Monaco · P. Fattori · C. Galletti

Department of Human and General Physiology,

University of Bologna, P.zza P.ta San Donato 2,

40127 Bologna, Italy

G. Króliczak

Human Neuroimaging and TMS Lab,

Department of Psychology, 1227 University of Oregon,

Eugene, OR 97403, USA

\section{Introduction}

The vast majority of kinematic studies of reaching have investigated actions toward external targets based on visual information; however, many everyday actions involve actions directed toward one's own body. These self-directed actions can be based on purely proprioceptive information, as in scratching an itch on an unseen shoulder, or based on a combination of proprioceptive and visual information, as in swatting a mosquito that is visible on the arm. Here, we asked how vision and proprioception contribute to the accuracy of reaching movements.

There is debate as to how information from various senses is combined. Some models suggest that sensory information is weighted optimally by the relative precision of each modality in both perceptual (Ernst and Banks 2002; Ernst and Bulthoff 2004) and sensorimotor tasks (van Beers et al. 1996, 1999). However, others have suggested that the relative contribution from different senses is context specific. For example, reaches to visual versus proprioceptive targets may rely more heavily on information about the hand's starting position coming from vision and proprioception, respectively (Sober and Sabes 2005; Sarlegna and Sainburg 2007, 2009). In addition, vision and proprioception may be differentially weighted in the planning of the movement vector and the transformation into joint angles, respectively (Sober and Sabes 2003). One suggested explanation for such results is that optimal sensory integration minimises transformations between different coordinate frames (Sober and Sabes 2005). That is, while visual information relies on extrinsic coordinates (i.e., using a reference frame outside the body), proprioceptive information relies on intrinsic coordinates (i.e., using a reference frame based on the body, such as joint angles). Because transformations between coordinate 
frames introduce noise (Soechting and Flanders 1989), sensory signals are said to be weighted so as to minimise such transformations.

If indeed coordinate transformations are undesirable in sensory integration, then different sensorimotor actions may be expected to vary in their weighting of information from multiple senses. Here, we investigated whether reaching movements would show a different reliance upon visual and proprioceptive information than saccadic eye movements (as investigated by an earlier project by Ren et al. 2006). Saccades require an eye movement of a certain amplitude in degrees of retinal angle and thus are more accurate for visual targets, in extrinsic coordinates, than proprioceptive targets, in intrinsic coordinates (Ren et al. 2006). In contrast, reaching requires the planning of both a movement vector in extrinsic coordinates and a combination of joint angles in intrinsic coordinates (Sober and Sabes 2003). As such, proprioceptive information may be more advantageous for reaching than saccades. Alternatively, some have suggested that all reaching movements, even those based on cues other than vision (e.g., audition), may be converted into an extrinsic (eye centred) reference frame (Cohen and Andersen 2000). If so, reaching accuracy may show a pattern similar to saccade accuracy, with poorer performance for combined vision + proprioception compared to vision alone.

We examined the accuracy of reaches to visual and proprioceptive targets using a paradigm from Ren et al. (2006) that has recently been used to study the accuracy of saccadic eye movements. They found that accuracy for saccades to remembered visual targets became degraded when proprioceptive information was added. Specifically, they had subjects make saccades to visual targets (a spot of light) or proprioceptive targets (a dowel held in the right hand, which was moved by the subject in active conditions or by the experimenter in passive conditions). In one set of conditions (visual memory), a brief (100 ms) visual stimulus at the target location was shown shortly $(1 \mathrm{~s})$ before the saccade was cued. In another set of conditions, no such visual preview was given, such that the saccade was based solely on current visual or proprioceptive information alone. They found that saccades overshot the targets, particularly in conditions when only proprioceptive information was available. More interestingly, in the conditions where a visual target was shown $1 \mathrm{~s}$ before the saccade, they found that proprioception + visual memory was less accurate than visual memory alone. This was particularly evident in the absolute errors but appeared to arise from both the constant error (particularly the overshoot) and the variable error (as reflected by the variability around the average endpoint). The authors suggested that proprioception is less accurate than vision and is thus weighted more weakly in the computation of end point. They further suggested that proprioception was not entirely ignored during the combined condition because the reliability of vision decayed over the delay interval.

Though several studies have examined the relative contribution of vision and proprioception to the accuracy of hand actions, the results have been equivocal, with some studies finding that the combination of proprioception and vision is worse than vision alone (Helms Tillery et al. 1991; Laufer and Hocherman 1998) and others finding that the combination is better than either sense alone (van Beers et al. 1996). The variability in findings is likely due to the wide variety of paradigms and experimental conditions. For example, different experiments have examined the contributions of different aspects of vision: vision of the external target per se (van Beers et al. 1998), vision of the workspace of the hand (van Beers et al. 1996), or vision of the starting position of the reaching hand (Desmurget et al. 1997; Lateiner and Sainburg 2003). Moreover, the reaches sometimes occurred after a delay of several seconds from the time that visual information was available (Helms Tillery et al. 1991; Laufer and Hocherman 1998). Proprioceptively guided actions, if they have similar properties as visually guided actions, may be expected to be less accurate after a delay than if the movements are made immediately (Westwood et al. 2001) and to recruit different neural mechanisms (Westwood and Goodale 2003; Goodale et al. 2004). In addition, the experimental conditions for proprioception also differed between studies. In some cases, proprioceptive information was available online (without delay) by having subjects reach with the index finger of one hand to the index finger of the opposite hand (van Beers et al. 1996; Desmurget et al. 1997). In other cases, subjects repeated arm movements to unseen locations and thus could only use remembered proprioceptive information (Helms Tillery et al. 1991; Laufer and Hocherman 1998; Chapman et al. 2001; Darling and Miller 1993). Although proprioceptive information about the dominant hand appears to decay more slowly than visual information (Desmurget et al. 1997; Chapman et al. 2001; Wann and Ibrahim 1992), it is not known whether immediate and delayed reaches to proprioceptive targets, like those to visual targets, rely on different neural mechanisms. Finally, in several of the studies, the final target position was indicated by the subject using a tool such as a stick or a laser pointer rather than the hand (van Beers et al. 1996; Helms Tillery et al. 1991; Chapman et al. 2001), making it difficult to dissociate errors in reaching accuracy per se versus errors arising from the transformations necessary to employ a tool. These methodological differences make it difficult to compare contributions of proprioception and vision in reaching movements to those reported by Ren et al. for saccadic movements.

Here we conducted two experiments to investigate how the variability of reaching endpoints in both amplitude and 
direction (which may utilise different sensory information; van Beers et al. 1998) is influenced by the availability of visual and/or proprioceptive information. The first experiment studied reaching accuracy using the same paradigm employed by Ren et al. to study saccade accuracy. That is, we had subjects use the right hand to reach to touch an LED mounted on top of a dowel (visual target) or an LED attached to the fingernail of subject's left index finger placed on top of the dowel. The subject's left finger could either be moved in place by the experimenter (Passively Positioned Body Target) or by the subject (Actively Positioned Body Target). The reach could be performed immediately after the visual target location was illuminated or as soon as the left finger reached the target location. Alternatively, a $1 \mathrm{~s}$ delay could be inserted between target positioning (visual target illumination and, in proprioceptive conditions, the movement of the left hand). The critical question was whether reaching accuracy, like saccade accuracy, would become worse when proprioceptive information was added to visual information, or whether, as some past studies have found, reaching accuracy would improve with the addition of proprioception. Importantly, unlike previous studies of reaching accuracy, in our first experiment we had subjects maintain fixation throughout each trial to ensure that reaching errors did not merely result from the errors in saccade planning that Ren et al. (2006) found. That is, had we permitted free viewing and found a similar pattern for reaching errors as was found with saccade errors, it may have been simply because the reach endpoint was drawn toward the saccadic endpoint, which as we know from Ren et al. shows more error when proprioception is available.

Unlike past studies that have looked at reaching accuracy (van Beers et al. 1996, 1998; Soechting and Flanders 1989; Helms Tillery et al. 1991; Laufer and Hocherman 1998), the paradigm employed in Experiment 1 (based on Ren et al.) enables a direct comparison between immediate and delayed actions. However, visual information is presented only briefly (100 ms), while proprioceptive information remains continuously available even $1 \mathrm{~s}$ after the left arm has moved. To phrase it another way, the reach is performed in visual open-loop conditions but proprioceptive closed-loop conditions. To compare the contribution of vision and proprioception to reaching accuracy under more similar conditions, and to investigate the contribution of visual feedback during the reach, we conducted a second experiment. In Experiment 2, subjects reached to either an external visual target or a proprioceptive target (the other hand held in place, i.e., closed-loop proprioception) with vision available throughout the trial (closed loop), vision available until movement onset (open loop), or no vision during either movement planning or execution. In all trials, a $2 \mathrm{~s}$ visual preview was presented $2 \mathrm{~s}$ before the movement cue. Unlike Experiment 1, subjects freely viewed the scene without a fixation point, enabling us to determine whether reach errors would in fact show the same pattern of errors as saccades when eye gaze could also guide reaching.

\section{Materials and methods: Experiment 1}

\section{Subjects}

Ten subjects ( 5 females, 5 males), aged 20-33 years, participated in this study. All participants were right-handed and had normal or corrected-to-normal vision. Participants gave their informed consent prior to testing. The experiment was approved by the Review Board for Non-Medical Research Involving Human Subjects at the University of Western Ontario.

\section{Procedure}

We replicated the study done by Ren et al. (2006) using the same paradigm and a similar apparatus (Fig. 1a). The key difference from their experiment was that while they studied the accuracy of saccades, we studied the accuracy of reaches. Subjects performed reaching movements with their right index finger either to an External Target or to a Body Target. Subjects sat in a dark room with the right hand placed at a starting position in front of them. Targets could be placed at one of eight locations equidistant $(10 \mathrm{~cm})$ from a central point. The apparatus consisted of a round turntable (radius $15 \mathrm{~cm}$, height $2 \mathrm{~cm}$ ) indented with a linear groove. The turntable could be rotated by the experimenter to align the groove along one of eight cardinal directions (Fig. 1a), at which point a lateral removable stopper was inserted to keep the turntable locked in place. The centre of the board was aligned to the subject's sagittal midline. A dowel (10 cm tall) was mounted upon a plate $(5 \times 5 \times 0.5 \mathrm{~cm})$ atop a guide pin with an inverted $\mathrm{T}$ shape that moved within the groove such that it could be slid along the groove without slipping out or tilting. Subjects were asked to maintain fixation on an LED located approximately $4 \mathrm{~cm}$ above the top of the dowel at the far position (position \#1 in Fig. 1a). Thus, the subject's head was tilted slightly downward such that the line of gaze was at an angle of approximately $45^{\circ}$ from the plane of the table.

Subjects reached to targets on the table top using different combinations of visual, proprioceptive, and memory information. In External Target conditions, the subject reached to touch a light-emitting diode (LED) attached to the top of a dowel that could be moved by the experimenter to one of the eight positions (Fig. 2, top row). In Passively 


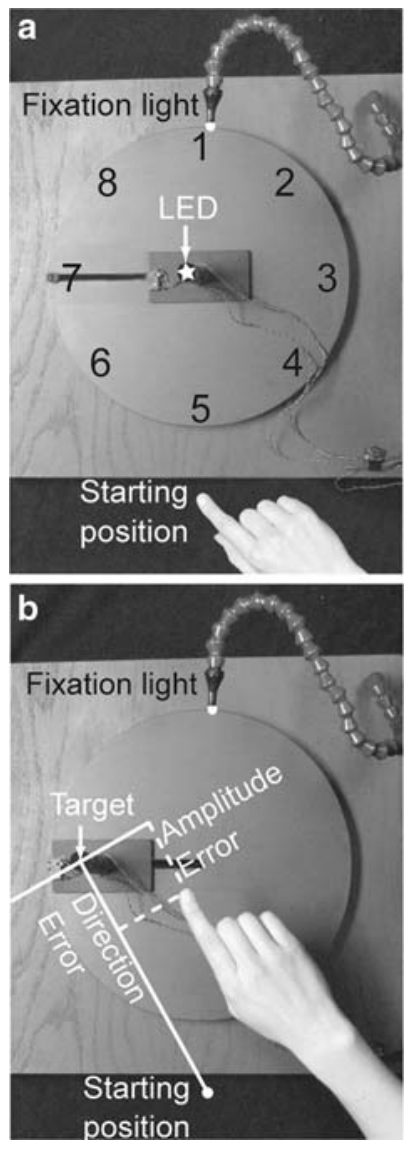

Fig. 1 Setup and derived measures-Experiment 1. a An overhead view of the subject's hand and the workspace in which subjects performed reaching movements toward one of the eight peripheral locations. The fixation light, at the end of a flexible stalk, was located approximately above the position number 1 . b Cartesian coordinate system used to derive the magnitude of the errors along two orthogonal axes. For each end location, the coordinate system was rotated in order to have the amplitude ( $y$ axis) correspond to the line linking the starting position to the target centre and the direction ( $x$ axis) correspond to the perpendicular side-to-side error

Positioned Body Target conditions, the subject reached to the left index finger placed at the top of the dowel, which was moved by the experimenter (Fig. 2, middle row). In the Actively Positioned Body Target conditions, the subject again reached to the left index finger placed at the top of the dowel, which this time was moved by the subject himself (Fig. 2, bottom row). Participants could feel whether or not their final reaching position made contact with the target LED or target finger, thus providing the potential for haptic feedback in all conditions. Although many past experiments have provided haptic feedback on all trials, or restricted it on all trials, we used a more natural paradigm in which feedback would be dependent upon accuracy. For example, in the real world, a person reaching to a moving target or a target in the dark will typically get feedback on successful trials, but not unsuccessful ones.
Fig. 2 Schematic of Experimental design and Timing-Experiment 1. Illustration of the timing of each trial in the No Visual Memory condition (left column) and Visual Memory condition (right column) for the External Target (top row), Passively Positioned Body Target (middle row) and Actively Positioned Body Target (bottom row). Even though the figures represent the workspace in the light, during the experiment all the lights were turned off allowing only the vision of the fixation light throughout the trials and the target LED (star) when it was on. For each combination of six conditions, two sequential images of the events are shown above a time line of the events. In the time lines, the solid line indicates when the target LED was on or off, the coarsely dashed line indicates the duration of the right hand reaching movement outward and inward, the finely dashed line indicates the movement of the subject's left hand into the target location, and the music note indicates the auditory cue. In the Visual Memory conditions the visual information was provided $1 \mathrm{~s}$ before the auditory tone that cued the movement with the reaching hand. In the No Visual Memory conditions the auditory tone occurred as soon as the LED was extinguished (in the External Target condition) or as soon as the subject's left hand was in place (in the Passively and Actively Positioned Body Target conditions)

In the Passively Positioned Body Target condition, we ensured that the subject's arm would not resist the movement of the dowel by the experimenter. Thus, we had subjects place the index finger atop the dowel (rather than having the hand hold the dowel, as in Ren et al. 2006). In addition, for both the Passively and Actively Positioned Body Target conditions, the left arm was strapped to an ergonomic forearm support (ERGOREST, Siilinjärvi, Finland) in order to allow the subject to completely relax the arm. The dowel was wedged into a hole in the forearm support and the left index finger was placed on the top of the dowel.

The three types of Targets (External Target, Passively Positioned Body Target and Actively Positioned Body Target) were crossed with the absence or presence of remembered visual information in No Visual Memory (Fig. 2, left column) and Visual Memory (Fig. 2, right column) conditions, respectively, to yield a total of six conditions. Participants started the movement upon hearing an auditory cue. In the No Visual Memory conditions, the cue occurred as soon as a $100 \mathrm{~ms}$ flash of light on the dowel was extinguished (in the External Target condition) or as soon as the subject's left hand was in place (in the Passively and Actively Positioned Body Target conditions). In the Visual Memory conditions, the cue was presented $1 \mathrm{~s}$ after the offset of a $100 \mathrm{~ms}$ flash of light at the target location (that is, at the top of the dowel in the External Target condition or on the subject's left fingertip in the Passively and Actively Positioned Body Target conditions). In the Passively and Actively Positioned Body Target conditions, the auditory cue (and in Visual Memory conditions the flash of light) was presented as soon as the dowel reached the end of the groove, which triggered a switch.

We included the Visual Memory conditions to allow a comparison between our results and results on saccades by 
No Visual Memory

험
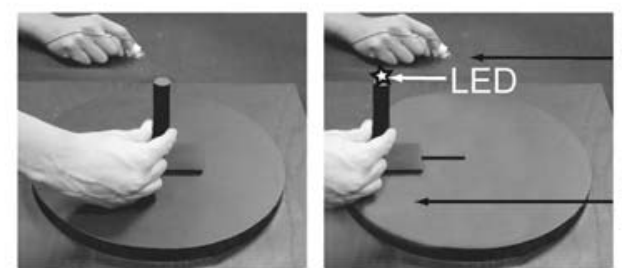

Subject's

reaching hand

Experimenter's

hand

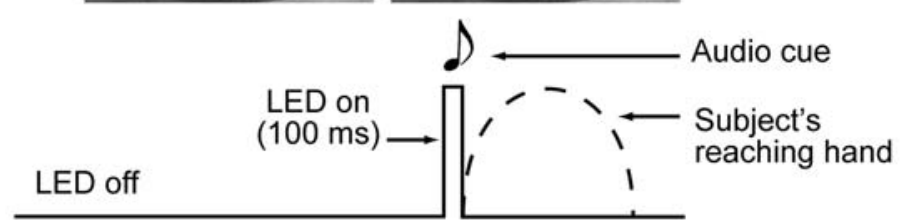

Time
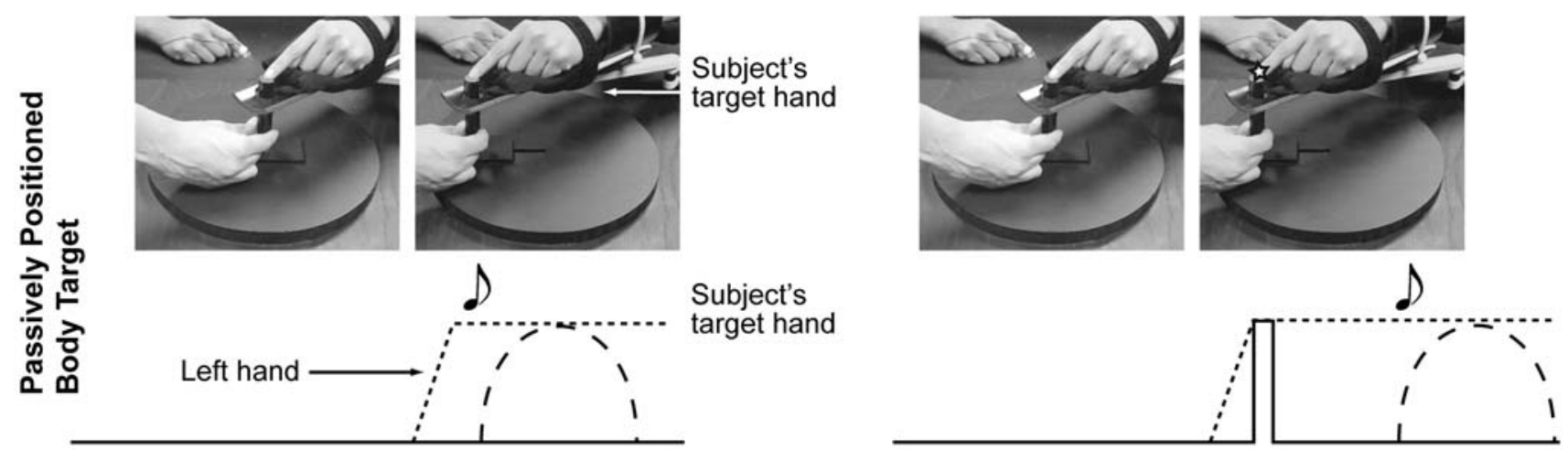

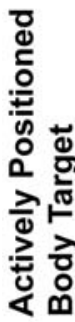
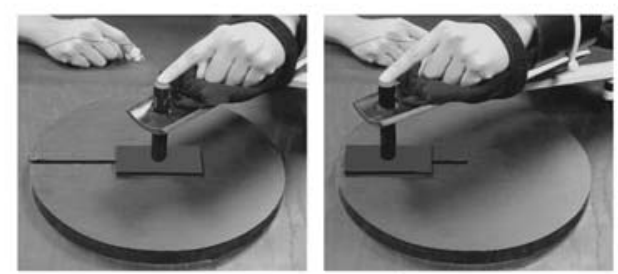

Visual Memory
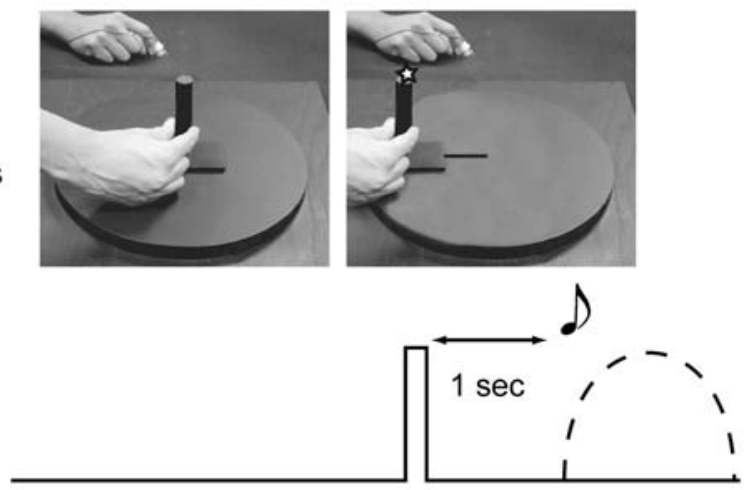

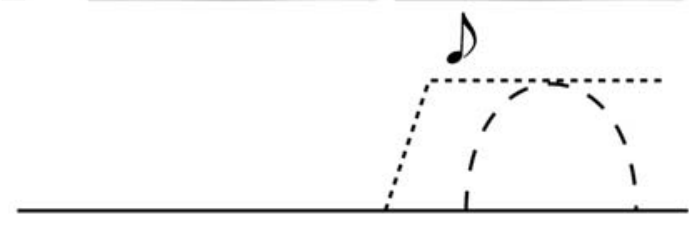

Ren et al. (2006) who also had 1 s delay. In addition, the inclusion of a delay condition allowed us to examine how sensory information degrades over short intervals. Previous research has shown that the visual system switches from online to memory-guided action as soon as vision is occluded (Westwood and Goodale 2003; Goodale et al. 2004; Heath et al. 2004). The proprioceptive system, however, may demonstrate a slower rate of decay over tens of seconds (Chapman et al. 2001; Wann and Ibrahim 1992; Desmurget et al. 2000). Although it would have been interesting to examine delays longer than $1 \mathrm{~s}$, because proprioceptive decay is so slow, we examined only brief delays to avoid making the testing sessions overly long. 
Subjects were tested in blocks for each of the six combinations of Target type (External Target, Passively Positioned Body Target, and Actively Positioned Body Target) and Visual Memory versus No Visual Memory. The order of blocks was randomized between subjects. Five reaching movements were made to each of the eight target positions in each of the six conditions, for a total of 240 trials for each subject. By collapsing across locations in the analysis, we obtained 40 trials per condition, providing a reasonable sample size. Before the actual experiment started, subjects performed two practice trials in each condition to get accustomed to the task. To prevent subjects from making reaches of a constant amplitude, each block included two additional trials with smaller movements $(5 \mathrm{~cm}$, with direction chosen randomly amongst the eight possibilities). These catch trials were not included in the analysis. The locations of the targets and catch trial stimuli were randomised to prevent subjects from simply repeating a hand trajectory in consecutive trials. Each of the six blocks took approximately $25 \mathrm{~min}$ to complete, with breaks of five minutes between blocks, such that each experiment lasted about three consecutive hours.

We recorded the kinematic parameters of an infrared emitting diode (IRED) located on the right reaching finger of the participant. An Optotrak 3020 motion-analysis system (Northern Digital, Waterloo, ON) recorded the position of the IRED at $100 \mathrm{~Hz}$. In offline analyses, movement velocity in the primary (anteroposterior) axis was used to define the start (faster than $20 \mathrm{~mm} / \mathrm{s}$ ) and the end (slower than $20 \mathrm{~mm} / \mathrm{s}$ ) of the movement.

To acquire the spatial coordinates $(X, Y)$ of the target locations, at the end of each experiment we performed two types of calibrations for each of the eight target locations: one for the External Target, in which participants placed the right index finger upon the illuminated LED and one for the Body Target, in which participants placed the right index finger upon the LED on the left fingertip. The endpoint of the IRED on the right index finger was taken as ideal endpoint and errors were quantified relative to this point. During calibration, subjects could optimise the final position with unlimited time and adjustments under full vision with free viewing; thus, these calibrations provide a more precise estimate of the ideal final position than the ballistic movements made during any of the experimental conditions.

At the end of each trial, the room was illuminated to prevent dark adaptation. No subject reported being able to see the hands during the experiment.

\section{Data analysis}

For two orthogonal directions of the reach, three dependent measures were derived: the absolute error, the constant error, and the variable error. The absolute error is the average distance of the endpoints from the target in the specified direction. The constant error (accuracy) reflects the bias in the average location of the endpoints taking into account the directionality of the error (for example, overshoot vs. undershoot in amplitude and right vs. leftward bias in direction). The variable error (precision) is a measure of the variability of the movements about the mean for an individual participant. The absolute error is dependent upon both constant and variable error and thus there is some redundancy in showing all three. Nevertheless, we chose to illustrate all three because the constant and variable error are valuable in interpreting the error patterns, while the absolute error gives an overall measure that has been used in past studies, including Ren et al. (2006).

Endpoint errors were calculated using a Cartesian coordinate system in the plane of the table. The $y$ axis was represented by the vector linking the starting position to the target centre (see Fig. 1b). This allowed us to distinguish two orthogonal components of reaching movements: the amplitude ( $y$ axis), defined as the near-far error, and the direction ( $x$ axis), defined as the side-to-side error. The amplitude is the distance between the endpoint and the $x$ axis of the coordinate system. The direction is the distance between the endpoint and the $y$ axis of the coordinate system. In both cases, the origin of the coordinate system is the endpoint determined through the calibration. On trials where the final endpoint of the reaching finger did not contact the target, the $x$ and $y$ endpoint positions were determined on the plane perpendicular to the top of the dowel.

To summarize the data qualitatively, we generated figures showing the patterns of errors for each of the spatial positions in each of the conditions (Fig. 3). In addition to showing the average endpoint across subjects, we also derived confidence ellipses to illustrate the within-subject variability of endpoints. Specifically, we computed the 95\% confidence intervals in the $x$ and $y$ directions for each subject, accounting for their covariance, and then displayed the average of these values. However, because of the need to collapse across locations to obtain larger sample sizes in each condition and because there was slight heterogeneity in the primary axis at each location, covariance was not taken into account in the statistical analyses.

To evaluate differences between conditions, planned statistical comparisons were performed in which subjects were treated as a random factor and data were collapsed across spatial locations. For each visual memory condition (No Visual Memory and Visual Memory) we ran a oneway ANOVA $(P<0.05)$ with repeated measures for each error type (absolute, constant or variable error) in each of the two dimensions (direction and amplitude). Where the ANOVA reached significance, post hoc $t$ tests were performed to clarify the nature of the differences (Bonferroni corrected for three comparisons). 
Fig. 3 Shape of within subjects 95\% confidence ellipses, shown for eight locations in each of the six conditions-Experiment 1. Actual target locations are indicated by the eight black squares around the circle where the lines intersect with the circle. The lines indicate the rotated axes joining the starting location to each of the target locations. The $x$ and $y$ axes (solid lines) are appropriate only for locations at the 12 o'clock and 6 o'clock locations. Dashed lines indicate the $y$ axes for other target locations; $x$ axes are orthogonal to those lines. Average endpoints for each location are indicated by eight black dots. The ellipses surrounding each black dot illustrate the within subjects 95\% confidence intervals centred on the mean of the endpoints
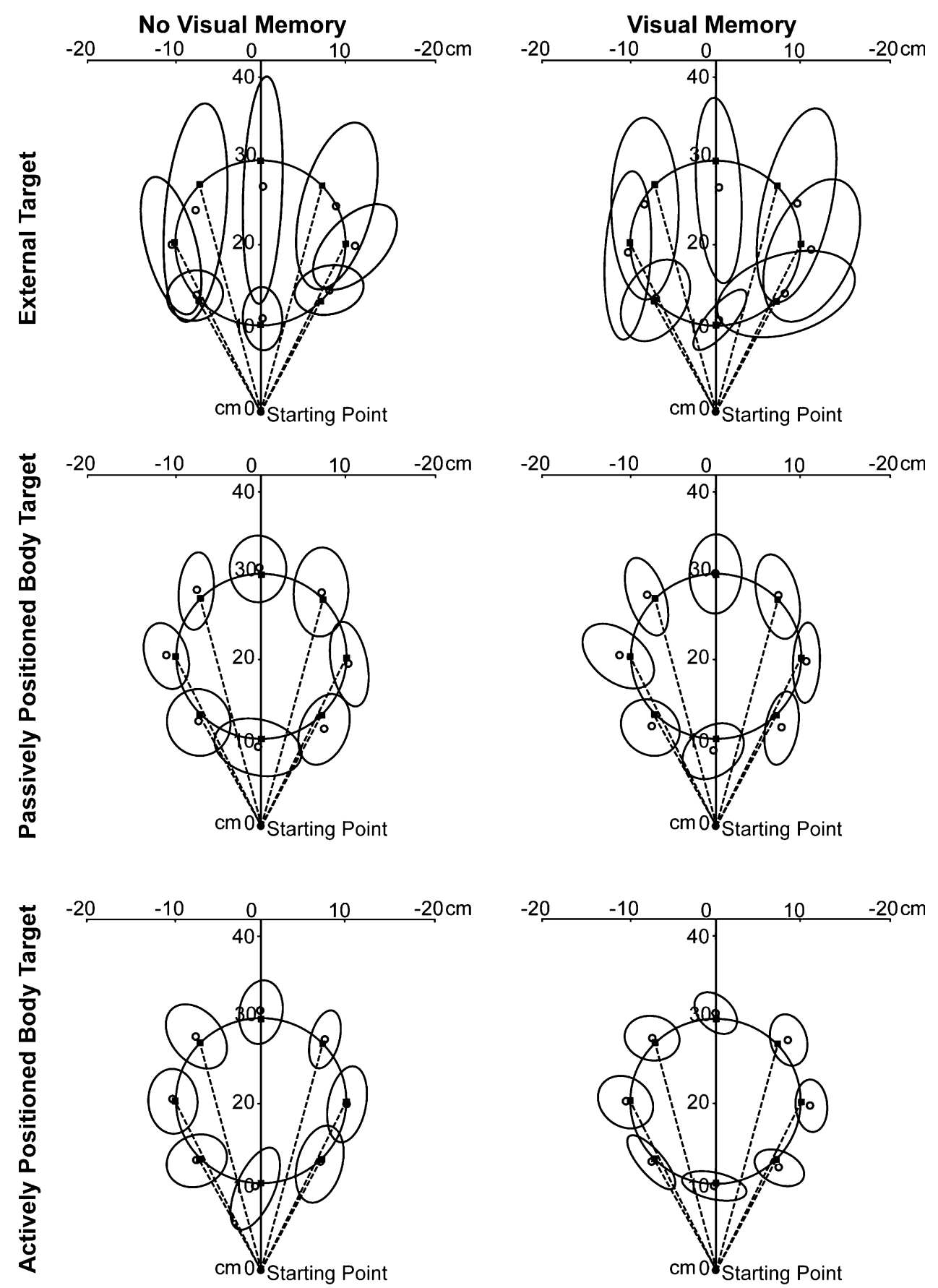

For each target type, we used paired sample $t$ tests to evaluate differences between No Visual Memory and Visual Memory conditions for each target type (Bonferroni corrected for three comparisons).

\section{Results: Experiment 1}

Figure 3 represents the average within subjects $95 \%$ confidence ellipses centred on the mean of the endpoints of all subjects for each condition. A qualitative analysis of Fig. 3 shows that, consistent with previous research (Messier and Kalaska 1997), in the External Target condition the ellipses tend to be elongated in amplitude. Errors are larger in the External Target condition than in the Passively and Actively Positioned Body Target conditions. The shape of the confidence ellipses in the External Target conditions varied over the workspace, with apparently larger amplitude errors for the further targets compared to the near targets and larger direction errors for the lateral locations compared to locations along the sagittal midline. There was also slight variability in the primary axis of elongation as a function of location. 
However, the small number of trials at each site in each subject precludes further analyses of locations.

\section{Absolute error}

For the direction axis, the External Target had larger errors than proprioceptive targets and it was affected by the addition of visual memory information while the proprioceptive targets were not (Fig. 4a). There were significant main effects of Target type in both No Visual Memory $[F(2,18)=9.187, P<0.005]$ and Visual Memory conditions $[F(2,18)=20.464, P<0.001]$. Post hoc paired sample $t$ tests revealed that in the No Visual Memory condition there were larger errors for External versus Actively Positioned Body Target $[t(9)=3.650, P<0.05]$ while the Passively Positioned Body Target condition did not differ significantly from either of the other two target types $[t(9)=2.494, P=0.103$ and $t(9)=2.277$,
$P=0.146]$. In the Visual Memory condition, the errors were larger for External Target versus Passively Positioned Body Target $[t(9)=5.089, P<0.005]$ and for External Target versus Actively Positioned Body Target $[t(9)=$ 4.843, $P<0.005$ ], while the two proprioceptive conditions did not differ significantly from each other $[t(9)=1.146$, $P=0.845]$. Moreover, while in the External Target condition the introduction of a delay significantly worsened the accuracy in Visual Memory compared to No Visual Memory condition [ $t(9)=5.023, P<0.005]$, there was no effect of delay for Passively $[t(9)=0.460, P=0.656]$ and Actively Positioned Body Targets $[t(9)=0.740$, $P=0.478]$.

For the amplitude axis (Fig. 4b), pointing accuracy was greatest in Actively Positioned Body Target, moderate in Passively Positioned Body Target and smallest in External Target condition. There were main effects of Target type in both No Visual Memory $[F(2,18)=8.708, P<0.005]$
Fig. 4 Graphs of the constant error (top row) absolute error (middle row) and variable error (bottom row) for direction (left column) and amplitude (right column) across all spatial locations-Experiment 1. The outcome of the $t$ tests is indicated by asterisks: $* P<0.05, * * P<0.01$, $* * * P<0.005, * * * * P<0.001$. Only significant results are indicated. The error bars represent the $95 \%$ confidence intervals, which indicate whether a value is significantly different from zero or not a

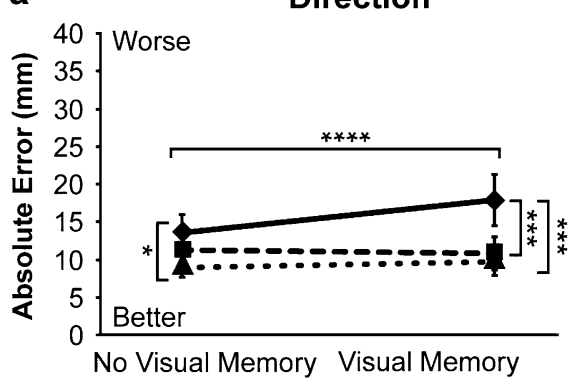

C

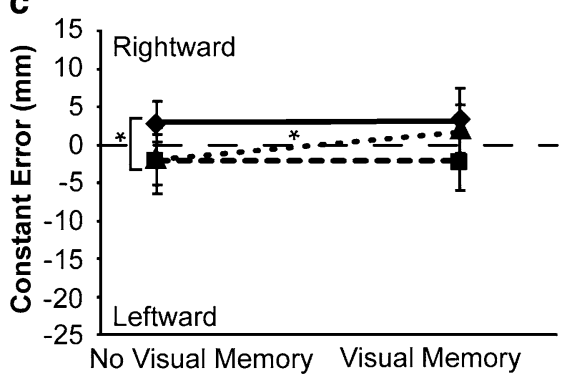

e

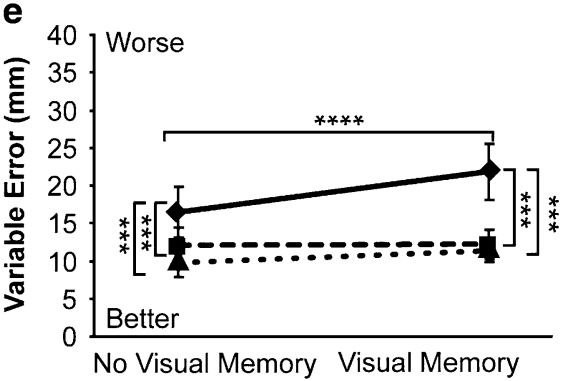

b Amplitude

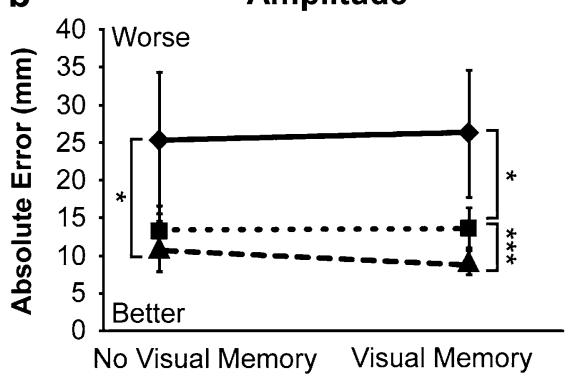

d

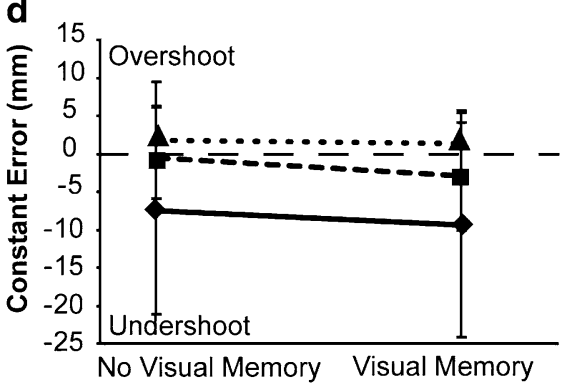

f

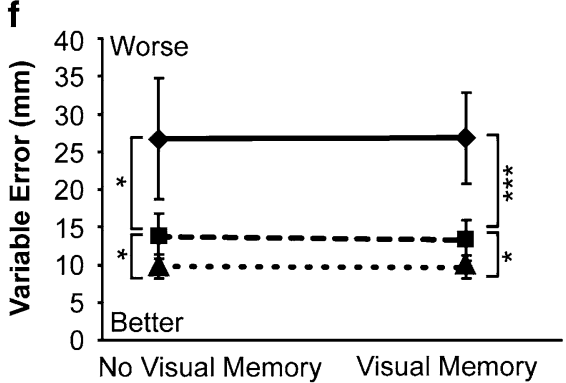


and Visual Memory conditions $[F(2,18)=19.063$, $P<0.001]$. Paired sample $t$ tests showed that in the No Visual Memory condition there was higher accuracy for Actively Positioned Body Target versus External Target $[t(9)=3.129, P<0.05]$ and there was a trend towards a difference between Passively Positioned Body Target versus External Target $[t(9)=2.803, P=0.062]$. In the Visual Memory condition, there was higher accuracy for Actively versus Passively Positioned Body Target $[t(9)=6.212$, $P<0.005]$ as well as for Passively Positioned Body target versus External Target $[t(9)=3.704, P<0.05]$. There were no significant differences between presence or absence of visual memory information in External Target $[t(9)=0.683, P=0.512]$, Passively Positioned Body Target $[t(9)=0.141, P=0.891]$ or Actively Positioned Body Target $[t(9)=1.926, P=0.086]$.

\section{Constant error}

Constant error was typically quite small and showed only a few differences from one condition to another.

For the direction axis, although the absolute value of the constant error was small for all three target types (Fig. 4c), it was larger and more rightward for the External Target compared to the Passive and Active Proprioception conditions (main effect of Target type for both Visual Memory $[F(2,18)=7.21, P<0.01]$ and No Visual Memory condition $[F(2,18)=4.291, P<0.05]$. In particular, in the No Visual Memory condition the error was more rightward in External Target versus Actively Positioned Body Target $[t(9)=3.976, P<0.05]$ and there was a trend towards significance in External Target versus Passively Positioned Body Target $[t(9)=2.672, P=0.077]$. In the Visual Memory condition, post doc $t$ tests did not reveal any significant effect between the three target types. In addition, while in External Target and Passively Positioned Body Target there was no difference between Visual Memory and No Visual Memory condition $[t(9)=0.086$, $P=0.934$ and $t(9)=0.034, P=0.974]$, in the Actively Positioned Body Target condition the errors were significantly more rightward in the Visual Memory versus No Visual memory condition $[t(9)=3.192, P=0.011]$.

For the amplitude axis (forward-backward), even though the absolute values of the errors were quite large, there were no significant differences between conditions because of the large variability (Fig. 4d). There were no main effects of target type in No Visual Memory $[F(2,18)=1.375$, $P=0.278]$ nor in Visual Memory condition $[F(2$, $18)=1.863, P=0.184]$ and there was no effect of remembered visual information in External Target $[t(9)=0.493$, $P=0.634]$, Passively Positioned $[t(9)=1.182, P=0.268]$ nor in Actively Positioned Body Target $[t(9)=0.185$, $P=0.857]$.

\section{Variable error}

The variable error term, which reflects the scatter about the mean location, shows a similar pattern as the absolute error.

For the direction axis, the External Target had larger variability than Passively and Actively Positioned Body Target and, moreover, was affected by the addition of visual memory information while the other two target types were not (Fig. 4e). There was a significant main effect of Target type in both No Visual Memory $[F(2,18)=19.422$, $P<0.001]$ and Visual Memory conditions $[F(2,18)=$ 28.197, $P<0.001]$. Post hoc paired sample $t$ tests indicated that in the No Visual Memory condition, there were larger errors for External Target versus Passively Positioned Body Target $[t(9)=4.502, P<0.005]$ as well as for External Target versus Actively Positioned Body Target $[t(10)=5.319, P<0.005]$ but not for Passively Positioned Body Target versus Actively Positioned Body Target $[t(9)=2.29, P=0.141]$. Similarly, in the Visual Memory conditions the errors were larger for External Target versus Passively Positioned Body Target $[t(9)=6.15, P<0.001]$ and for External Target versus Actively Positioned Body Target $[t(9)=5.173$, $P<0.005]$ but not for Passively Positioned Body Target versus Actively Positioned Body Target $[t(9)=1.046$, $P=0.968]$. In addition, only the External Target condition was affected by the absence or presence of visual information, while the Passively and Actively Positioned Body Target conditions were not. Specifically, in the External Target condition there were smaller errors for No Visual Memory versus Visual Memory [ $t(9)=4.902, P<0.005]$. In contrast, in Passively Positioned Body Target, there was no difference between Visual Memory versus No Visual Memory $[t(9)=0.053, P=0.959]$; similarly in the Actively Positioned Body Target condition there was also no significant difference between Visual Memory versus No Visual Memory $[t(9)=1.328, P=0.217]$.

For the amplitude axis, pointing precision was greatest in the Actively Positioned Body Target condition, moderate in the Passively Positioned Body Target condition and the worst in the External Target condition (Fig. 4f). Specifically, there was a significant main effect of Target type in both No Visual Memory $[F(2,18)=20.139, P<0.005]$ and Visual Memory conditions $[F(2,18)=33.847$, $P<0.001]$. Paired sample $t$ tests showed that in both No Visual Memory and Visual Memory conditions there were larger errors for the External Target versus Passively Positioned Body Target $[t(9)=3.686, P<0.05$ and $t(9)=5.049, \quad P<0.005]$ and for Passively Positioned Body Target versus Actively Positioned Body Target $[t(9)=3.692, P<0.05$ and $t(9)=3.305, P<0.05]$. In addition, there was no effect of remembered visual 
information in External Target $[t(9)=0.053, P=0.959]$, Passively Positioned Body Target $[t(9)=0.721, P=0.489]$ nor Actively Positioned Body Target condition $[t(9)=0.142$, $P=0.89]$.

\section{Introduction: Experiment 2}

As mentioned above, the finding of Experiment 1 that the accuracy of reaching was better in proprioception only conditions than in vision only conditions, could be due to the fact that visual performance was suboptimal because the visual targets were always in peripheral vision. Alternatively or additionally, in both Passively and Actively Positioned Body Target conditions subjects had online proprioceptive information during both movement planning and execution, while in the External Target condition, they had a brief (100 ms) visual information only during movement planning but not during movement execution. If the subjects had had the visual targets in central view and the same online visual information also during movement execution, perhaps the performance would have been as good as in the Body Target conditions. To test this hypothesis, in Experiment 2 we had subjects reaching, with free viewing, to either their own left fingertip (Passively Positioned Body Target) or to an External Target with varying degrees of visual information available. Of course, it is impossible to reach toward a target when neither proprioceptive nor visual information is available; therefore, in all conditions we added a $2 \mathrm{~s}$ preview followed by a $2 \mathrm{~s}$ delay before the reach was cued. Thus, we had six combinations in a $2 \times 3$ design with Passively Positioned Body Target and External Target crossed with three levels of vision: no online visual information at the time of action (Preview Only), visual information until movement onset (Preview + Brief Vision), or online visual information until the end of the movement (Preview + Full Vision). If accuracy differences between vision and proprioception arose from differences in feedback, reaching to an External target in a visual closed-loop condition (External Target, Preview + Full Vision) would be at least as accurate as reaching to a Body target in a proprioceptive closed-loop condition (Passively Positioned Body Target, Preview + No Vision). Moreover, based on the results of van Beers et al. (1998), we expected that directional accuracy would be more affected by the degree of visual information than would amplitude accuracy, regardless of proprioceptive information. Because this design already had six conditions and we observed few differences between Passive and Active conditions in Experiment 1, we decided to test only Passive conditions. Note that the effects of Passively Positioned Body Target information on amplitude and direction were not investigated by van Beers et al.

\section{Materials and methods: Experiment 2}

Subjects

Twelve subjects (3 females, 9 males), aged 23-35 years, participated in this study. As in Experiment 1, they were right-handed, had normal or corrected-to-normal vision and gave informed consent.

Procedure

Subjects performed reaching movements with the right index finger either to an External Target or to a Passively Positioned Body target (see Fig. 5a, b). The External Target was a movable target placed on the workspace by the experimenter, while the Passively Positioned Body target was represented by the subject's left fingertip that was passively moved and put in place by the experimenter. It is worth emphasising that because the arm movements for Passively Positioned Body Target positioning were passive movements the information available to subjects regarding the position of their arm was exclusively derived from proprioceptive information and not from an efference copy (corollary discharge) related to the arm movement. Subjects always started with their reaching finger located on a button directly in front of them.

The amount of visual information was varied in three conditions: Preview + Full Vision, Preview + Brief Vision, and Preview Only (See Fig. 5c). In each visual condition the subject had a $2 \mathrm{~s}$ preview followed by a $2 \mathrm{~s}$ delay, after which an auditory cue informed the subject to start the movement. In the Preview + Full Vision condition, the vision of the workspace was available until the end of the movement; in the Preview + Brief Vision condition, the workspace was visible until movement onset as indicated by the release of the starting button; in the Preview Only condition, subjects performed the reaching movement to a target without vision during planning and execution phases of the movement. The visual events were controlled using a set of PLATO liquid crystal display (LCD) goggles (Translucent Technologies, Toronto, ON, Canada), which could be made opaque or transparent. When the goggles were open, during the preview and eventually during the action, the workspace and the hand in the starting position were visible and participants freely viewed the display without a fixation point. The visual feedback of the initial hand position was important to prevent drift in proprioception during visual occlusion (Brown et al. 2003). At the end of each trial participants returned with the right hand to the felt button position with goggles closed.

Subjects were comfortably seated facing a flat table upon which they could perform reaching movements with 

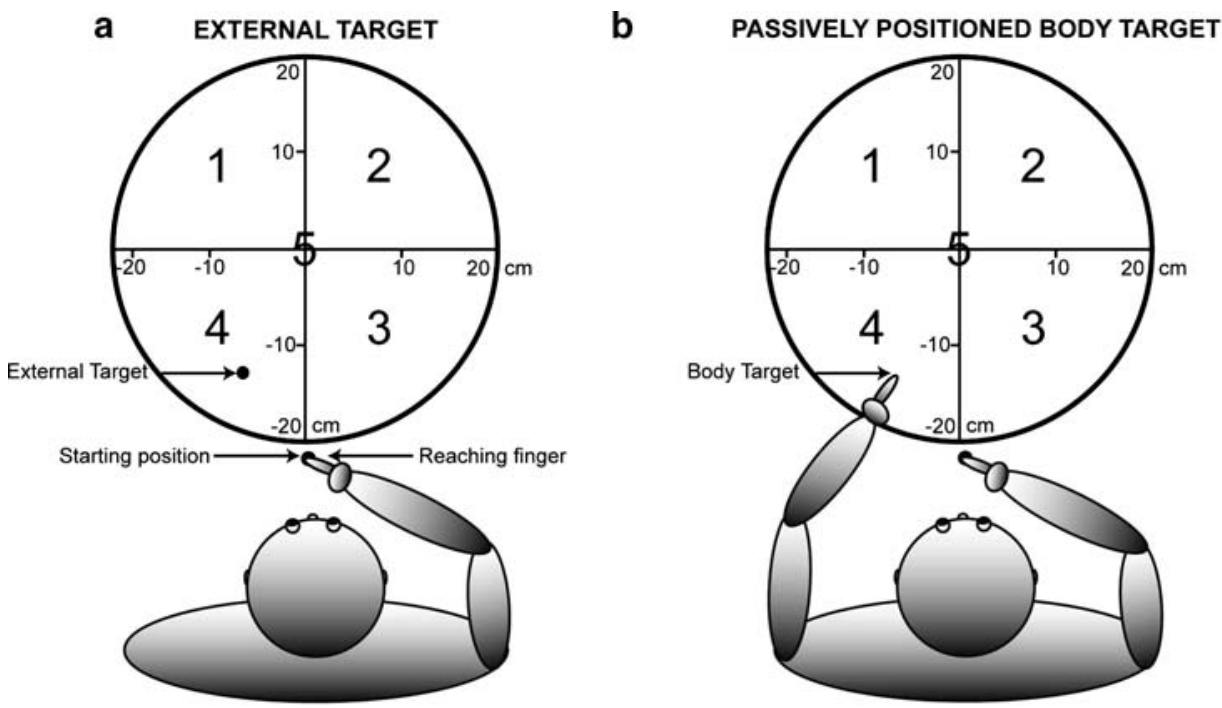

C TIMING

External Target

\section{Passively Positioned Body Target}

\section{Preview Only}
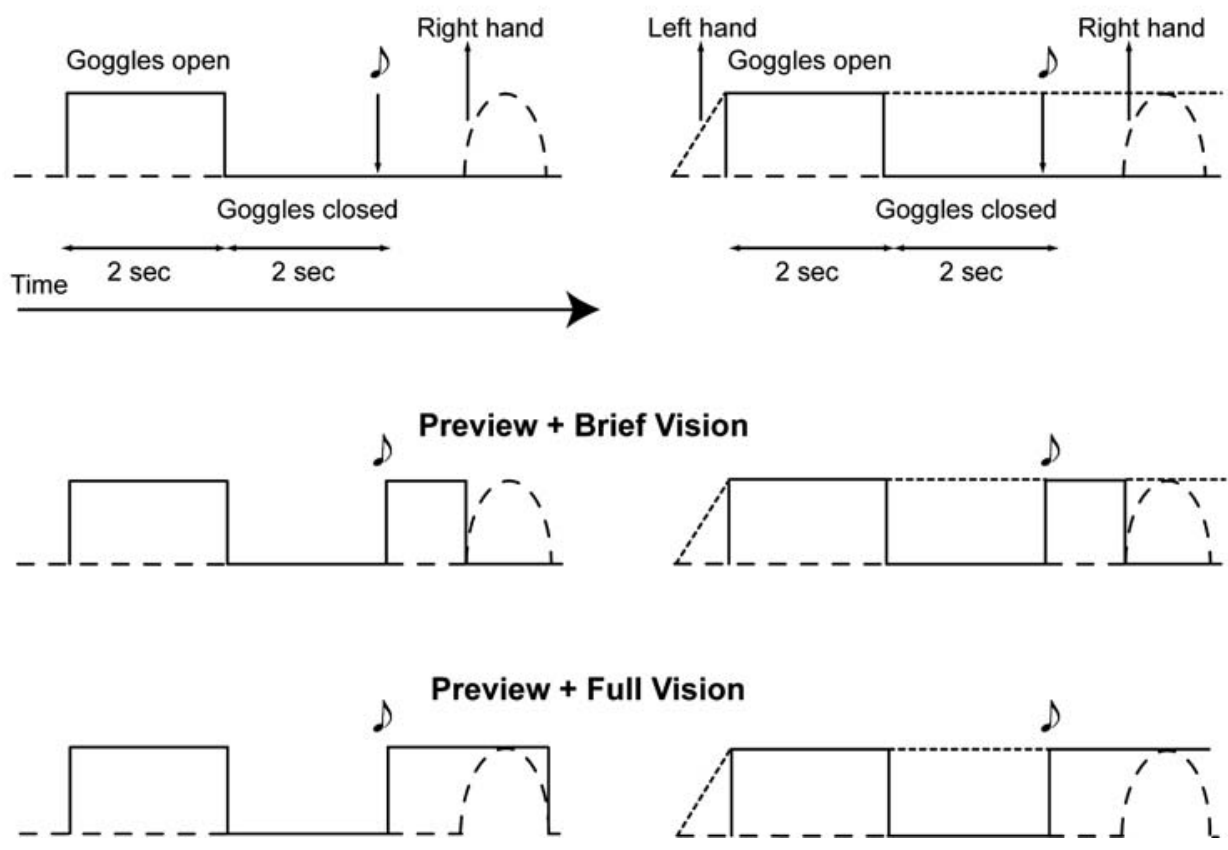

Fig. 5 Schematic of Experimental Setup and Timing-Experiment 2. a An overhead view of the subject and the workspace in the External Target condition in which subjects performed reaching movements toward a small white cylinder (indicated by the black dot). The workspace is represented by the circle, and the numbers $(1,2,3,4$, and 5) correspond to the five general zones where the target could be placed by the experimenter. b An overhead view of the Body Target condition. Subjects performed reaching movements towards their own left fingertip after the experimenter had moved the arm to position the fingertip at the desired endpoint. c Illustration of the timing of each trial in the External Target condition (left) and in the Passively Positioned Body Target condition (right). As shown by the solid line indicating when the goggles were open, in each condition there was a
$2 \mathrm{~s}$ visual preview of the target followed by a $2 \mathrm{~s}$ delay without vision. An auditory go-signal occurred after the delay to cue the movement of the right reaching hand, represented by the coarsely dashed line. In the Body Target condition, the left hand, represented by the finely dashed line, was moved by the experimenter before the preview period and remained in place until the end of the trial. During the execution of the movement there were three visual conditions: in the Preview Only condition, no vision was available during planning and execution of the movement and only memory was available; in the Preview + Brief Vision condition, vision was available until movement onset; in the Preview + Full Vision condition, vision was available until movement end 
their right hand to either the External or Passively Positioned Body Target. On each trial, one of two black wooden boards $(18.5 \mathrm{~cm} \times 7.5 \mathrm{~cm} \times 1 \mathrm{~cm}) \quad$ was placed horizontally on the table by the experimenter in order to indicate the position to be reached. On one of the boards, a cylindrical block (radius $0.50 \mathrm{~cm}$, height $1 \mathrm{~cm}$ ) was mounted to provide the External Target. On the other board, a nail was mounted to provide a location on which the subject's left index finger rested. The table was covered with black cloth in order to reduce the noise and the slipping of the targets on the table during the tasks. In addition, the underside of the two boards was also covered in black cloth and the boards were painted black to reduce their visibility against the table. The cylinder that served as the External Target was covered with white cloth tape, similar to the shape and the contrast of the fingertip, and was attached on the superior surface of the board.

The workspace was represented by a circle (radius $20 \mathrm{~cm}$ ) divided into five zones- the centre of the circle and four quadrants-in which the target was placed by the experimenter at the beginning of each trial. The locations of the targets were not fixed: targets could be placed anywhere within each quadrant by the experimenter. The five zones were equally represented in each condition and were randomised in order to avoid spatial learning. The starting position was between the subject and the board, $3.5 \mathrm{~cm}$ away from the board and in line with the midsagittal plane of the subject. Subjects were asked not to move their trunk with respect to the table during the experiment; small movements, however, could not be prevented.

Experimental trials were organised into six blocks: two target conditions (External Target, Passively Positioned Body Target) by three visual conditions (Preview + Full Vision, Preview + Brief Vision, Preview Only). The order of blocks was randomized between subjects. Three reaching movements were made to each target position (total five zones) in each of the six conditions, for a total of 90 trials for each subject. By collapsing across locations in the analysis, we obtained 15 trials per condition, providing a reasonable sample size. Before the actual experiment started, subjects performed three practice trials in each condition to get accustomed to the task. Each of the six blocks took less than $10 \mathrm{~min}$ and subjects could take breaks between blocks as desired. Including setup time, the experiment took about an hour and a half to complete and was performed in a single session.

\section{Data analysis}

Using the same procedure as Experiment 1, we recorded the kinematic parameters of three infrared emitting diodes (IREDs) located on the right reaching finger of the participant, on the External Target and on the Passively
Positioned Body Target (left fingertip) at $300 \mathrm{~Hz}$. Again, amplitude and direction were computed as in Experiment 1 (See Fig. 1b).

Data were analysed separately for each combination of error (constant error or variable error) and the axis of error (direction or amplitude). For each combination, we computed a 2 (target type) $\times 3$ (levels of visual information) repeated measures ANOVA. For completeness, all graphs show data for all conditions; however, when interactions did not reach statistical significance, we have also summarised differences related to main effects in subsidiary panels to the right within each full plot (Fig. 7).

We also evaluated how the variable error for amplitude and direction compared between the closed-loop proprioception condition (Passively Positioned Body Target with a Preview Only) and the closed-loop vision condition (External Target with a Preview + Full Vision). We computed a 2 (target type) $\times 2$ (axis) ANOVA (Fig. 8).

\section{Results: Experiment 2}

Figure 6 represents the within subjects $95 \%$ confidence ellipses centred on the mean of the endpoints for each condition. It is evident that even though the average of the endpoint movements (black dot) is always very close to the target position (intersection of axes), the size and shape of the ellipses differ between conditions. Note that the leftright error ( $x$ diameter) was similar for External Target and Passively Positioned Body Target across each of the visual conditions. In contrast, the forward-back error ( $y$ diameter) was larger for the External Target, particularly when vision was limited.

\section{Constant error}

The absolute values of the constant error term, which reflects the bias in the average location of the endpoints, were quite small and there were few differences between conditions.

For the direction axis, the constant errors were small and there were no significant differences between conditions (Fig. 7a). There were no main effects of target type $[F(1,11)=2.2, P=0.17]$ or visual information $[F(2,22)=$ 2.4, $P=0.12]$ and no interaction $[F(2,22)=0.08$, $P=0.93]$. However, the average direction errors were significantly different from zero (right side of Fig. 8a, $P<0.05)$.

For the amplitude axis, although the constant error was small for both target types (Fig. 7b), it was larger for the Passively Positioned Body Target compared to the External Target [main effect of target type, $F(1,11)=5.6$, $P<0.05]$. The main effect of visual information showed a 


\section{External Target Passively Positioned Body Target}
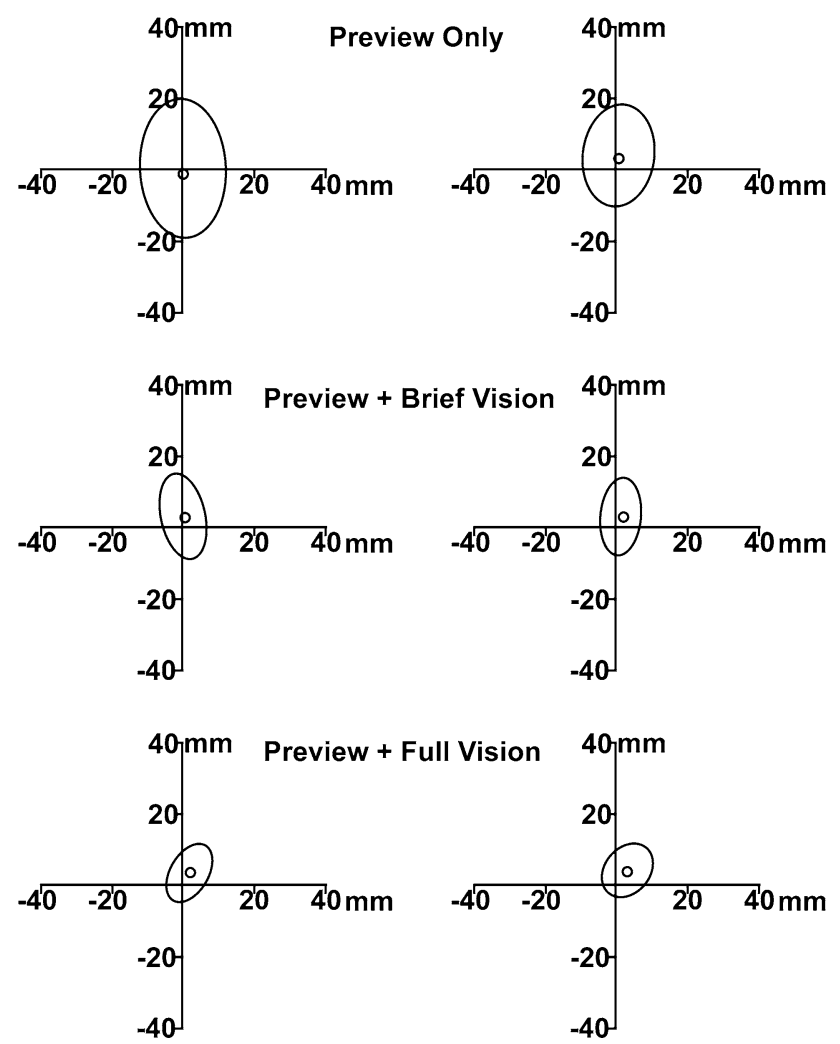

Fig. 6 Shape of within subjects $95 \%$ confidence ellipses in six conditions-Experiment 2. Each plot shows the mean of the endpoints relative to the actual target location at the origin of the axes, which represent the direction ( $x$ axis) and amplitude ( $y$ axis) of the movement. Data shown are collapsed across all locations in the five zones. The mean location of the endpoints (constant error) is shown with a black dot. The ovals represent the within subjects $95 \%$ confidence ellipses centred on the mean of the endpoints

trend toward significance $[F(2,22)=3.1, P=0.065]$ but there was no interaction $[F(2,22)=2.0, P=0.15]$. The average amplitude error for the Passively Positioned Body Target was significantly greater than zero $(P<0.005)$; whereas the average amplitude error for the External Target was not $(P=0.18)$.

\section{Variable error}

The variable error term, which reflects the scatter about the mean location, shows a more complex and interesting pattern.

For the direction axis, both the Body and External Targets showed an improvement in accuracy with increasing visual information (Fig. 7c). Errors were smaller for the Passively Positioned Body Target than the External Target only in the Preview Only condition. Specifically, there were significant main effects of target type $[F(1,11)=$ $30.1, P<0.05]$ and visual information $[F(2,22)=78.5$, $P<0.001], \quad$ as well as a significant interaction $[F(2,22)=4.5, P<0.05]$. Post hoc paired sample $t$ tests indicated that both the External Target and the Passively Positioned Body Target were affected by the amount of visual information. Specifically, for the External Target, there were larger errors for the Preview Only versus Preview + Brief Vision $[t(11)=6.8, P<0.001]$ and for the Preview + Brief Vision versus Preview + Full Vision $[t(11)=7.2$, $P<0.001]$. Similarly, for the Passively Positioned Body Target, there were also larger errors for the Preview Only versus Preview + Brief Vision $[t(11)=4.6, P<0.01]$ and for the Preview + Brief Vision versus Preview + Full Vision $[t(11)=4.4, P<0.01]$. In addition, the Passively Positioned Body Target had smaller errors than the External Target only for Preview Only $[t(11)=0.3, P<0.01]$ but not for Preview + Brief Vision $[t(11)=1.9, P=0.08]$ or Preview + Full Vision $[t(11)=0.4, P=0.70]$.

For the amplitude axis, pointing variability to the External Target improved considerably with the addition of visual information; whereas, the Passively Positioned Body Target showed much less of an improvement (Fig. 7d). This pattern was also reflected by the finding that the improvement in variability for the Body versus External Target was greatest for the Preview Only condition, moderate for the Preview + Brief Vision, and weakest for Preview + Full Vision. Specifically, there were significant main effects of target type $[F(1,11)=42.1, P<0.001]$ and visual information $[F(2,22)=74.4, P<0.001]$, as well as a significant interaction $[F(2,22)=21.8, P<0.001]$. Post hoc paired sample $t$ tests indicated that the External Target was affected by the amount of visual information, with larger errors for Preview Only versus Preview + Brief Vision $[t(11)=6.0$, $P<0.001]$ and for Preview + Brief Vision versus Preview + Full Vision $[t(11)=10.1, P<0.001]$. In contrast, for the Passively Positioned Body Target, only the difference between Preview Only versus Preview + Full Vision reached significance $[t(11)=3.8, P<0.01]$, while the differences between Preview Only versus Preview + Brief Vision $[t(11)=1.6, P=0.40]$ and Preview + Brief Vision versus Preview + Full Vision $[t(11)=2.3, P=0.10]$ did not. In addition, the Passively Positioned Body Target had smaller errors than the External Target for both Preview Only $[t(11)=6.7, \quad P<0.001]$ and Preview + Brief Vision $[t(11)=3.0, P<0.05]$ but not for Preview + Full Vision $[t(11)=1.9, P<0.08]$.

As shown in Fig. 8, the ANOVA comparing closed-loop proprioception (Passively Positioned Body Target with a Preview Only) with closed-loop vision (External Target with a Preview + Full Vision) for Direction and Amplitude found a main effect of target type $[F(1,11)=15.05$, $P<0.005]$ and a significant interaction $[F(1,11)=42.9$, $P<0.001]$. The variability in direction was larger for closed-loop proprioception than for closed-loop vision $[t(11)=11.541, P<0.001]$. In contrast, the variability in amplitude was not significantly different $[t(11)=0.36$, 
Fig. 7 Graphs of the constant error (top row) and variable error (bottom row) for direction (left column) and amplitude (right column)—Experiment 2. For the constant error plots, in which there was no interaction, statistically significant differences related to the main effects are represented to the right of the condition-specific means. For the variable error plots, in which interactions were significant, the outcome of post hoc $t$ tests is indicated by asterisks. Conventions are as in Fig. 4
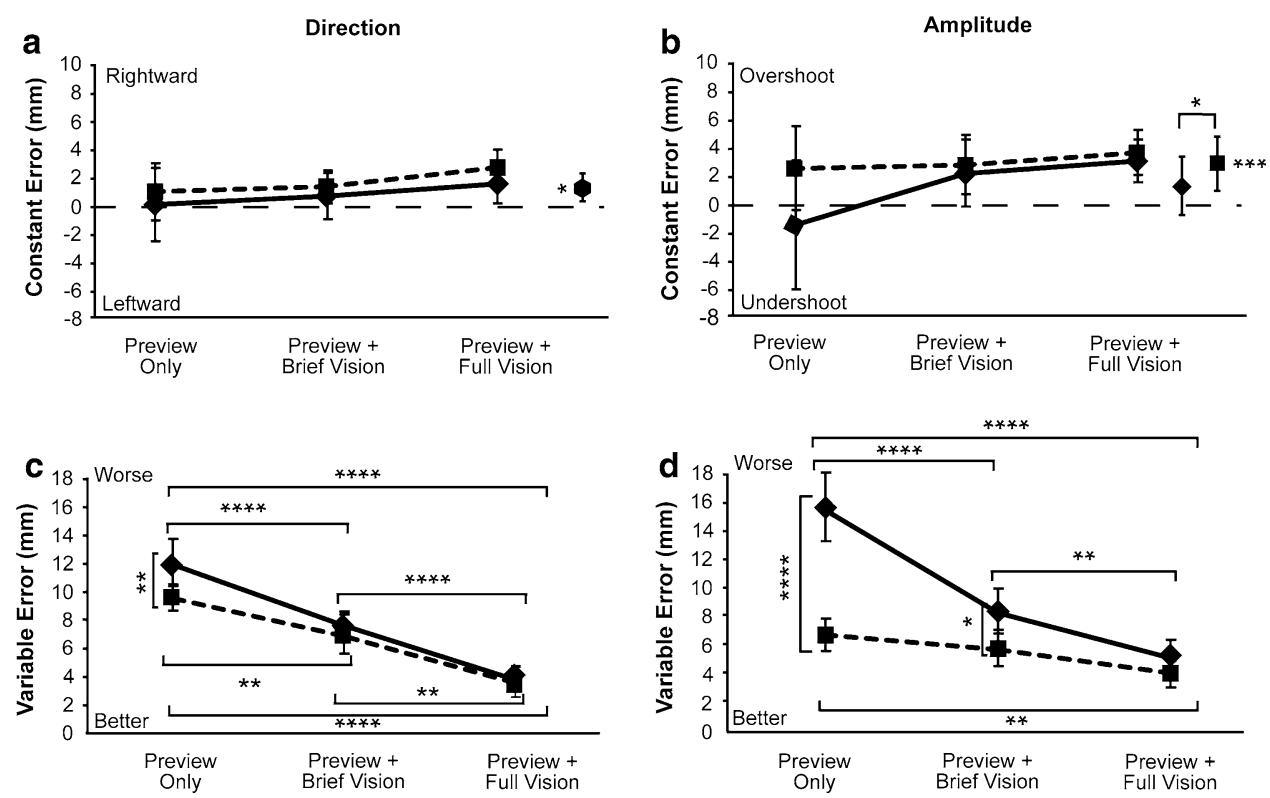

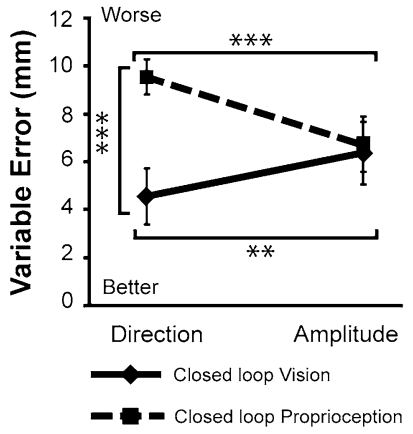

Fig. 8 Graphs of the visual and proprioceptive closed-loop conditions for amplitude and direction-Experiment 2. A subset of data points from Fig. 7 have been replotted to facilitate evaluation of a new contrast. Conventions are as in Fig. 4

$P=0.73]$. Furthermore, while for the closed-loop visual target, the amplitude error was larger than the directional error $[t(11)=4.964, P<0.001]$, for the closed-loop proprioception target, the directional error was larger than the amplitude error $[t(11)=3.936, P<0.005]$. These comparisons reinforce the relative importance of vision for direction and proprioception for amplitude.

\section{Discussion}

Utility of proprioceptive information for reaching versus saccades

Results of both experiments show that proprioceptive information is of greater utility for reaching movements than saccades (as investigated by Ren et al. 2006). Our first experiment demonstrated that subjects were more accurate and more precise when reaching to the finger of the opposite hand than when reaching to an external visual target, as shown by results of variable and absolute error. This is in contrast to saccades which were less accurate and less precise for proprioceptive than visual targets (Ren et al. 2006). Moreover, unlike saccades (Ren et al. 2006), reach precision is improved rather than impaired by the addition of somatosensory information to visual information. In our second experiment, we further investigated whether the benefits of proprioception over vision in the first experiment depended on the fact that targets appeared in peripheral vision (making localisation less precise) or the fact that visual but not proprioceptive feedback was limited. We found that proprioceptive information conferred an additional benefit when vision was limited even with free viewing and continuous visual feedback.

The greater reliance of reaches on proprioceptive information and saccades on visual information suggests that the neural systems subserving the two actions weight sensory information differently. That is, while the saccade system may have a poor internal model of limb coordinates (Ren et al. 2006), the reach system has a more accurate model, even when proprioceptive information is arriving from the opposite arm and hand. Differences in the way sensory information is utilised in the two sensorimotor systems are likely related to the coordinate systems in which they work. Eye movements are planned in eye centred (extrinsic) reference frame while there is growing 
evidence that hand movements are planned in mixed hand and eye-reference frame (Pesaran et al. 2006; Batista et al. 2007; Marzocchi et al. 2008; McGuire and Sabes 2009) Therefore, for the saccade system, the addition of proprioception to vision requires additional coordinate transformations; whereas, reaches already require transformations between multiple coordinate frames. Although there are methodological differences, our results are consistent with other studies suggesting that sensory weighting is goal dependent (Sober and Sabes 2005; Sarlegna and Sainburg 2007; Sarlegna and Sainburg 2009; Sober and Sabes 2003). However, while those studies manipulated the proprioceptive information of either the reaching hand at the starting location (visual-proprioceptive mismatch) (Sarlegna and Sainburg 2007; Sober and Sabes 2003) or the target hand (veridical or shifted position) (Sober and Sabes 2003, 2005), we manipulated the sensory modality of target presentation (visual, proprioceptive or both). In addition, the similarity of our paradigm to that used for saccades (Ren et al. 2006) enables comparisons between reaches and saccades.

Although there were minor methodological differences between Experiment 1 and Ren et al. (2006), we do not believe these can account for the different findings with reaching versus saccades. First, Ren et al. had subjects holding a short dowel between the finger and thumb; whereas, we had subjects place the index finger atop a taller dowel. Although a recent study finds that precision and accuracy for reaches to proprioceptive targets are better for targets with high tactile receptor density (Brown et al. 2008), in both our study and that of Ren et al. reaches were made near the index fingertip, which has high tactile resolution. Second, in the No Visual Memory conditions of our first experiment, the reach was cued as soon as the dowel stopped, unlike Ren et al. where there was a delay of several seconds. However, as noted earlier, proprioception decays slowly (Chapman et al. 2001; Wann and Ibrahim 1992; Desmurget et al. 2000). Third, in Experiment 1, gaze was fixed off-centre above the furthest target location; whereas, in Ren et al. gaze began in the centre of the display and then moved toward the target. As such, our targets may have had a slightly greater displacement in depth relative to fixation than those in Ren et al. However, it is unlikely that the dissociable patterns observed for reaches and saccades are due to this minor difference. Additional uncertainty regarding target depth from vision may be expected to increase errors in the amplitude dimension, yet we found that the addition of proprioception to visual information improved precision for both amplitude and direction. Moreover, in Experiment 2, where fixation was not constrained, we still observed an advantage for proprioception. Fourth, with reaches, but not saccades in the dark, there was the potential for haptic feedback from the reaching hand. If the hand missed the target, no such feedback would be received (except to know that the target was missed). Thus, it is possible that conditions with higher success on initial trials may have incurred more valuable feedback. However, since the locations of the targets were randomised between blocks of conditions in both experiments, it's unlikely that subjects could have used information about the position of a target in a given successful trial for the subsequent one. In addition, if the difference in feedback had been a major factor in our experiment, we would have expected a difference between Closed-Loop Vision, during which visual feedback was provided at each trial, and Closed-Loop Proprioception, during which visual feedback was not available and subjects only had a proprioceptive feedback when they reached the correct target position. Indeed, there is no difference for variable errors in amplitude between these two conditions. Moreover, some of our findings are consistent with previous research where feedback was not provided on any trial (van Beers et al. 1996, 1998) or was given on each trial (Sober and Sabes 2005).

Our main goal was to investigate differences between sensory conditions. Although location was varied to avoid predictability of the target, we did not systematically examine the effects of location upon error patterns due to the small number of trials per location per condition. Nevertheless our conclusions regarding reach precision across conditions would be unlikely to change if location were factored in. We conducted an analysis on the data from Experiment 1 which collapsed errors across Near, Medium and Far locations or Left, Middle and Right locations to gain enough trials (15) per location per condition. For variable error in both direction and amplitude, this analysis revealed only effects of target type with no main effects of location or interactions between location and condition. Thus, our conclusions regarding reach precision appear to generalise across the range of locations tested.

Differences in encoding amplitude and direction

Our results extend past research suggesting that vision is more important for determining reach direction, while proprioception is more important for determining reach amplitude (van Beers et al. 1996, 1998, 1999; Soechting and Flanders 1989). One classic experiment by van Beers et al. (1998) mathematically derived the contributions of the left hand, right hand, and vision to direction and amplitude based on three conditions that each included two of the components (left hand reach to a visual target, right hand reach to a visual target, and left hand reach to right hand location). Although this approach was valuable, it assumes that the contribution of each component is the 
same regardless of the combination (e.g., the contribution of the right hand is the same regardless of whether it is the target or the acting hand). In contrast, our experiments directly measured the contribution of the two senses alone and in combination.

\section{Coding of amplitude using proprioception}

Although precision was better for Body Targets than External Targets along both axes, precision was better for Actively Positioned versus Passively Positioned Body Targets only along the amplitude axis. While van Beers (van Beers et al. 1996, 1998) examined only Actively Positioned Body Target, our experiments also studied the effects of Passively Positioned Body Target. Neurophysiological data have shown that even with just passive positioning of the arm, more neurons in primary somatosensory cortex (S1) are more sensitive to the movement amplitude than direction (Tillery et al. 1996). We found that precision in the amplitude of reaching movements was better for Actively than Passively Positioned Body Targets (Experiment 1), whereas there was no difference for the direction of movements. Others have found an advantage for Active versus Passive conditions (Paillard and Brouchon 1968; Paillard and Brouchon 1974; Adamovich et al. 1998). However, in one study (Adamovich et al. 1998), variable errors were significantly smaller for Active than Passive conditions only for direction but not amplitude (although their Fig. 2a indicates a trend in the expected direction for amplitude as well). The discrepancy between their study and ours may be due to methodological differences, including the plane of target locations and the type of movements (in their case, participants reached to a remembered location where the reaching arm had previously been positioned).

In both Passively and Actively Positioned Body Target conditions, participants had transient and tonic proprioceptive signals from tendons, joints and muscles due to the displacement of the left arm. An additional corollary discharge of the motor command was sent to the brain in the Actively Positioned Body Target condition. Thus, our results indicate that the corollary discharge of the motor command is particularly useful in coding the distance of the finger from our own body (vs. the direction). These signals are more precise in the active than in the passive placement of the arm since they allow the specification of the joint angles during the generation of the movement.

For both direction and amplitude, accuracy and precision were unaffected by the introduction of a $1 \mathrm{~s}$ delay. Although transient proprioceptive signals and corollary discharge would have decayed over this interval, tonic proprioceptive signals regarding joint position continued to be available. These results suggest that transient proprioceptive signals and corollary discharge are resistant to decay over brief intervals.

\section{Coding of direction using vision}

Our results also reinforce the importance of vision for coding direction. This is most striking in Experiment 2, where we found that the precision for direction was much better during closed-loop vision than closed-loop proprioception. Moreover, with closed-loop vision the precision for direction was much better than for amplitude. These effects were found even when participants looked directly at the targets. Thus, even though the addition of proprioceptive information impairs saccade accuracy and precision, this impairment does not propagate to reaching even when gaze is maintained upon the target.

The relative utility of vision for direction versus amplitude likely results from the computations necessary to derive the two components. The visual system codes vertical and horizontal directions with considerable fidelity (e.g., retinotopic mapping experiments). However, the coding of depth is much more complicated, utilising not just the retinal location of the stimulus but also the vergence status of the two eyes, binocular disparity information, and monocular depth cues. Vergence is particularly important. Indeed, recent work from our lab suggests that a reach-selective region of the human brain codes vergence distance, which may be critical for determining reach amplitude (Quinlan and Culham 2007; Culham et al. 2008). Like proprioception of limb position, vergence status could be derived from corollary discharge as well as phasic and tonic proprioceptive signals (but from the eye muscles rather than joints). In short, the computation of target location in depth requires an integration of visual and position signals; whereas, the computation of target location in direction depends only on retinal position.

Although we found that proprioceptive signals were resistant to brief delays after visual presentation of a target, visual signals were degraded for direction when no proprioceptive information was available, even with only a $1 \mathrm{~s}$ delay (Experiment 1). Past studies have shown visual information decays quickly (as reviewed in (Goodale et al. 2004). There has been some debate as to the time course of the decay, with some suggesting that even very brief delays have dramatic effects (Westwood et al. 2001; Westwood and Goodale 2003; Heath et al. 2004) and others suggesting that the critical detriment of visual memory happens within the first second after vision occlusion (Rossetti 1998). Regardless, the delays we used of $1 \mathrm{~s}$ (Experiment 1) and $2 \mathrm{~s}$ (Experiment 2) should have been sufficient to lead to a visual detriment, as our results from the two experiments indicate.

The results of Experiment 1 show that only direction suffers from the introduction of a delay. To our knowledge, 
this is a novel finding. Given that the coding of amplitude depends on vergence information, one parsimonious explanation for our results is that the proprioceptive signals from the eye muscles, like those from the arm, are more resistant to decay that the retinal position signals. Alternatively, this may be a "floor effect" in that amplitude coding is poor even without a delay and may not become noticeably worse with brief delays.

\section{Summary}

In summary, our experiments contributed several novel findings to the literature on sensory integration during sensorimotor tasks. First and most importantly, we have shown that reaches rely more heavily on proprioceptive information than saccades. Although a wide variety of past studies investigated sensory integration in reaching, the methods were very heterogeneous and did not compare reaching to saccades. Our experiments allow a direct comparison with saccades studied using the same paradigm (Ren et al. 2006). These results indicate that the reaching system has a better internal model for limb positions (even when using the limb coordinates from one arm to guide reaches with the other arm) than does the saccade system. Second, our results clarify the relative utility of proprioception and vision for determining reach amplitude and direction, respectively. Extending past findings that passive proprioception is particularly valuable for determining reach amplitude, we now find that active proprioception confers even greater benefits, but only for reach amplitude. Third, our results are the first to show that the decay of visual information is limited to direction but not amplitude, perhaps because proprioceptive vergence signals, like arm proprioception, are resilient to brief delays. While our results are highly consistent with a variety of past studies of reaching arguing that sensory integration is context specific, they show critical differences between two major sensorimotor systems (saccades and reaches) and the factors that determine reach accuracy in amplitude and direction.

Acknowledgments The authors thank John Kalaska, Yvonne Wong, Richard Lee and Giacomo Placenti for helpful discussions and Haitao Yang for technical support. This research was funded by an operating grant from the Natural Science and Engineering Research Council of Canada (Grant \# 249877-02 RGPIN) to Jody C. Culham.

\section{References}

Adamovich SV, Berkinblit MB, Fookson O, Poizner H (1998) Pointing in 3D space to remembered targets. I. Kinesthetic versus visual target presentation. J Neurophysiol 79:2833-2846

Batista AP, Santhanam G, Yu BM, Ryu SI, Afshar A, Shenoy KV (2007) Reference frames for reach planning in macaque dorsal premotor cortex. J Neurophysiol 98:966-983. doi:10.1152/ jn.00421.2006

Brown LE, Rosenbaum DA, Sainburg RL (2003) Limb position drift: implications for control of posture and movement. J Neurophysiol 90:3105-3118. doi:10.1152/jn.00013.2003

Brown LE, Morrissey BF, Goodale MA (2008) Vision in the palm of your hand. Neuropsychologia. doi:10.1016/j.neuropsychologia. 2008.11.021

Chapman CD, Heath MD, Westwood DA, Roy EA (2001) Memory for kinesthetically defined target location: evidence for manual asymmetries. Brain Cogn 46:62-66

Cohen YE, Andersen RA (2000) Reaches to sounds encoded in an eye-centered reference frame. Neuron 27:647-652

Culham JC, Gallivan J, Cavina-Pratesi C, Quinlan DJ (2008) fMRI investigations of reaching and ego space in human superior parieto-occipital cortex. In: Klatzky RL, Behrmann M, MacWhinney B (eds) Embodiment, Ego-space and Action. Psychology Press, New York, pp 247-274

Darling WG, Miller GF (1993) Transformations between visual and kinesthetic coordinate systems in reaches to remembered object locations and orientations. Exp Brain Res 93:534-547

Desmurget M, Rossetti Y, Jordan M, Meckler C, Prablanc C (1997) Viewing the hand prior to movement improves accuracy of pointing performed toward the unseen contralateral hand. Exp Brain Res 115:180-186

Desmurget M, Vindras P, Grea H, Viviani P, Grafton ST (2000) Proprioception does not quickly drift during visual occlusion. Exp Brain Res 134:363-377

Ernst MO, Banks MS (2002) Humans integrate visual and haptic information in a statistically optimal fashion. Nature 415:429 433. doi: $10.1038 / 415429$ a

Ernst MO, Bulthoff $\mathrm{HH}$ (2004) Merging the senses into a robust percept. Trends Cogn Sci 8:162-169. doi:10.1016/j.tics. 2004.02.002

Goodale MA, Westwood DA, Milner AD (2004) Two distinct modes of control for object-directed action. Prog Brain Res 144:131144

Heath M, Westwood DA, Binsted G (2004) The control of memoryguided reaching movements in peripersonal space. Mot Control 8:76-106

Helms Tillery SI, Flanders M, Soechting JF (1991) A coordinate system for the synthesis of visual and kinesthetic information. J Neurosci 11:770

Lateiner JE, Sainburg RL (2003) Differential contributions of vision and proprioception to movement accuracy. Exp Brain Res 151:446-454. doi:10.1007/s00221-003-1503-8

Laufer Y, Hocherman S (1998) Visual and kinesthetic control of goaldirected movements to visually and kinesthetically presented targets. Percept Mot Skills 86:1375-1391

Marzocchi N, Breveglieri R, Galletti C, Fattori P (2008) Reaching activity in parietal area V6A of macaque: eye influence on arm activity or retinocentric coding of reaching movements? Eur J Neurosci 27:775-789. doi:10.1111/j.1460-9568.2008.06021.x

McGuire LM, Sabes PN (2009) Sensory transformations and the use of multiple reference frames for reach planning. Nat Neurosci 12:1056-1061. doi:10.1038/nn.2357

Messier J, Kalaska JF (1997) Differential effect of task conditions on errors of direction and extent of reaching movements. Exp Brain Res 115:469-478

Paillard J, Brouchon M (1968) Active and passive movements in the calibration of position sense. In: Freedman SJ (ed) The Neuropsychology of Spatially Oriented Behavior. Dorsey Press, Homewood III, pp 37-55

Paillard J, Brouchon M (1974) A proprioceptive contribution to the spatial encoding of position cues for ballistic movements. Brain Res 71:273-284 
Pesaran B, Nelson MJ, Andersen RA (2006) Dorsal premotor neurons encode the relative position of the hand, eye, and goal during reach planning. Neuron 51:125-134. doi:10.1016/j.neuron. 2006.05.025

Quinlan DJ, Culham JC (2007) fMRI reveals a preference for near viewing in the human parieto-occipital cortex. Neuroimage 36:167-187. doi:10.1016/j.neuroimage.2007.02.029

Ren L, Khan AZ, Blohm G, Henriques DY, Sergio LE, Crawford JD (2006) Proprioceptive guidance of saccades in eye-hand coordination. J Neurophysiol 96:1464-1477. doi:10.1152/jn.01012. 2005

Rossetti Y (1998) Implicit short-lived motor representations of space in brain damaged and healthy subjects. Conscious Cogn 7:520 558. doi:10.1006/ccog. 1998.0370

Sarlegna FR, Sainburg RL (2007) The effect of target modality on visual and proprioceptive contributions to the control of movement distance. Exp Brain Res 176:267-280. doi:10.1007/ s00221-006-0613-5

Sarlegna FR, Sainburg RL (2009) The roles of vision and proprioception in the planning of reaching movements. Adv Exp Med Biol 629:317-335

Sober SJ, Sabes PN (2003) Multisensory integration during motor planning. J Neurosci 23:6982-6992
Sober SJ, Sabes PN (2005) Flexible strategies for sensory integration during motor planning. Nat Neurosci 8:490-497. doi:10.1038/nn1427

Soechting JF, Flanders M (1989) Sensorimotor representations for pointing to targets in three-dimensional space. J Neurophysiol 62:582-594

Tillery SI, Soechting JF, Ebner TJ (1996) Somatosensory cortical activity in relation to arm posture: nonuniform spatial tuning. J Neurophysiol 76:2423-2438

van Beers RJ, Sittig AC, Denier van der Gon JJ (1996) How humans combine simultaneous proprioceptive and visual position information. Exp Brain Res 111:253-261

van Beers RJ, Sittig AC, Denier van der Gon JJ JJ (1998) The precision of proprioceptive position sense. Exp Brain Res 122:367-377

van Beers RJ, Sittig AC, Gon JJ (1999) Integration of proprioceptive and visual position-information: An experimentally supported model. J Neurophysiol 81:1355-1364

Wann JP, Ibrahim SF (1992) Does limb proprioception drift? Exp Brain Res 91:162-166

Westwood DA, Goodale MA (2003) Perceptual illusion and the realtime control of action. Spat Vis 16:243-254

Westwood DA, Heath M, Roy EA (2001) The accuracy of reaching movements in brief delay conditions. Can J Exp Psychol 55:304-310 